\title{
Criminologie
}

\section{La tolérance des juges à la fraude fiscale : un inconscient d'institution}

\section{Judicial attitudes towards tax fraud: the unconscious at work in institutions}

\section{La tolerancia de los jueces al fraude fiscal : un inconsciente institucional}

\section{Alexis Spire et Katia Weidenfeld}

Volume 49, numéro 1, printemps 2016

Quand le crime économique contribue au développement des sciences sociales

URI : https://id.erudit.org/iderudit/1036195ar

DOI : https://doi.org/10.7202/1036195ar

Aller au sommaire du numéro

Éditeur(s)

Les Presses de l’Université de Montréal

ISSN

0316-0041 (imprimé)

1492-1367 (numérique)

Découvrir la revue

Citer cet article

Spire, A. \& Weidenfeld, K. (2016). La tolérance des juges à la fraude fiscale : un inconscient d'institution. Criminologie, 49(1), 79-98.

https://doi.org/10.7202/1036195ar
Résumé de l'article

Cette contribution vise à montrer que la régulation de la fraude fiscale par l'institution judiciaire traduit une tolérance, en partie inconsciente, des acteurs qui en sont chargés. Pour en analyser les mécanismes, on s'intéresse aux discours et aux pratiques des procureurs et des magistrats chargés d'assurer le suivi pénal des dossiers que leur transmet l'administration fiscale. Tous se disent convaincus de la nécessité de lutter contre cette forme de délinquance en col blanc, mais s'abstiennent d'utiliser tous les moyens dont ils disposent pour que la pénalisation de la fraude fiscale débouche sur des sanctions effectives. Il en découle une indulgence qui contraste avec la posture d'intransigeance adoptée par l'institution judiciaire dans d'autres domaines. Pour comprendre les ressorts de cette impunité fiscale, nous proposons une approche qui tient compte de la singularité de ce délit et du profil social de ceux qui le commettent, en considérant à la fois les contraintes institutionnelles qui s'imposent aux magistrats et les représentations qui les rendent acceptables. 


\title{
La tolérance des juges à la fraude fiscale : un inconscient d'institution
}

\author{
Alexis Spire ${ }^{1}$ \\ IRIS (CNRS-EHESS) \\ alexis.spire@gmail.com \\ Katia Weidenfeld \\ École nationale des chartes \\ katia.weidenfeld@enc.sorbonne.fr
}

RÉSUMÉ - Cette contribution vise à montrer que la régulation de la fraude fiscale par l'institution judiciaire traduit une tolérance, en partie inconsciente, des acteurs qui en sont chargés. Pour en analyser les mécanismes, on s'intéresse aux discours et aux pratiques des procureurs et des magistrats chargés d'assurer le suivi pénal des dossiers que leur transmet l'administration fiscale. Tous se disent convaincus de la nécessité de lutter contre cette forme de délinquance en col blanc, mais s'abstiennent d'utiliser tous les moyens dont ils disposent pour que la pénalisation de la fraude fiscale débouche sur des sanctions effectives. Il en découle une indulgence qui contraste avec la posture d'intransigeance adoptée par l'institution judiciaire dans d'autres domaines. Pour comprendre les ressorts de cette impunité fiscale, nous proposons une approche qui tient compte de la singularité de ce délit et du profil social de ceux qui le commettent, en considérant à la fois les contraintes institutionnelles qui s'imposent aux magistrats et les représentations qui les rendent acceptables.

MOTS CLÉS - Fraude fiscale, délinquance en col blanc, sociologie du droit, institution judiciaire, normes.

\section{Introduction}

Le thème de la fraude fiscale a très longtemps occupé une place relativement marginale dans les travaux sur la délinquance en col blanc. Dans ses textes programmatiques, Sutherland (1983) préconise de s'intéresser aux délits des entreprises et de ceux qui les dirigent, en prenant comme

1. Institut de recherche interdisciplinaire sur les enjeux sociaux - IRIS (CNRS-EHESS), 190-198, avenue de France, 75244 Paris CEDEX 13. 
exemples le non-respect des règles commerciales, les entraves à la concurrence, les actes de corruption, la violation de normes alimentaires ou pharmaceutiques... La transgression des règles fiscales y apparaît de façon incidente et a longtemps été négligée par les criminologues qui se sont inscrits dans son sillage. La publicité donnée aux formes légales d'évitement de l'impôt (Weidenfeld, 2010) a incité l'administration à labelliser la déviance comme relevant essentiellement de l'optimisation (legal avoidance), catégorie nettement distinguée de la fraude (evasion) à laquelle ne renvoyaient que les manquements les moins sophistiqués (refus pur et simple de déclarer ou de payer l'impôt).

Depuis les années 1980, les manuels consacrés à la délinquance en col blanc font une place à ce délit (Coleman, 1994), mais ils l'envisagent le plus souvent sous l'angle des motivations de ceux qui évitent l'impôt (Shover et Hochstetler, 2005 ; Simpson, 2002) ou des opportunités dont ils disposent pour minimiser leur contribution (Leroy, 2010). Les études sur la répression de la fraude privilégient le plus souvent ses liens avec le civisme fiscal: un angle d'approche récurrent consiste ainsi à se demander si la probabilité d'être sanctionné et la gravité de la peine encourue influent sur la propension à éviter l'impôt (Braithwaite, 1989; Klepper et Nagin, 1989; Murphy, 2008).

L'objectif de cet article n'est pas d'apporter une nouvelle contribution à ces débats. Il s'inscrit davantage dans la perspective des travaux qui soulignent les obstacles à une véritable régulation pénale de la déviance fiscale (Cook, 1989; McBarnet, 1991). L'enjeu ici est de mettre en lumière les mécanismes sociaux et institutionnels qui conduisent les juges à ne pas sanctionner la fraude fiscale comme un délit à part entière.

En France, l'évitement de l'impôt est depuis la fin des années 2000 au cœur du débat public; les acteurs chargés d'en réguler les excès affichent une réprobation unanime. Pourtant, si l'on en juge par le nombre des poursuites (moins de mille par an) et par les sanctions effectivement prononcées (dans presque $80 \%$ des cas, de la prison avec sursis), force est de constater que perdure une forme d'impunité. D'un côté, les magistrats du siège et du parquet, lorsqu'on les interroge, fustigent cette forme d'incivisme. De l'autre, les condamnations prononcées par les tribunaux judiciaires contre les fraudeurs fiscaux conduisent à des sanctions invisibles. Cette contradiction peut s'analyser comme un inconscient d'institution: l'impunité fiscale est en effet inscrite à la fois dans les procédures régulant la pénalisation de 
la fraude fiscale (notamment dans les modalités de sélection des dossiers, de conduite des enquêtes et de choix des peines) et dans les croyances et les évidences généralement partagées par les agents qui y travaillent. La force de cet inconscient d'institution est de puiser sa légitimité dans une histoire longue de l'impunité fiscale qu'on peut faire remonter à l'Ancien Régime (Spire et Weidenfeld, 2015). Comme dans d'autres sphères étatiques, la longévité de cet inconscient d'institution n'exclut pas que certains acteurs puissent en prendre conscience et s'en libérer (Bourdieu, 1980). C'est à la faveur d'un sursaut des magistrats, en septembre 2013, que Jérôme Cahuzac, alors ministre du Budget, a été mis en examen pour blanchiment de fraude fiscale. Ce mouvement a trouvé un écho dans la mise en cause, à l'initiative des juges d'instruction, de plusieurs banques (UBS ou HSBC). Mais ces spectaculaires coups d'éclat renseignés par des journalistes ou des salariés lanceurs d'alerte ne doivent pas occulter l'écrasante majorité des autres affaires de fraude fiscale. La réalité quotidienne du traitement de cette infraction par les magistrats reste prisonnière d'une mécanique institutionnelle qui rechigne à l'appréhender comme un véritable délit.

Pour comprendre la mansuétude des juges, il faut d'abord restituer la place de la fraude fiscale dans le champ judiciaire et rappeler le rôle des filtres successifs qui interviennent en amont de toute intervention de la justice. Dans la plupart des cas, les acteurs de l'institution judiciaire se contentent d'entériner des constats déjà opérés par l'administration fiscale qui dispose du monopole dans le déclenchement de la procédure pénale. Persuadés que leur investissement ne sera pas efficient, les magistrats n'utilisent pas - ou très peu - les moyens dont ils disposent pour compléter les investigations réalisées par l'administration fiscale (I). Mais l'impunité ne résulte pas directement de ce renoncement: la plupart des contribuables poursuivis sont en effet condamnés. Les peines sont toutefois singulièrement plus clémentes que celles qui sont prises à l'encontre de la délinquance conventionnelle ou même d'autres délinquances économiques (escroquerie ou fraude sociale, par exemple). Cette mansuétude traduit, en partie, la place particulière qu'occupe la déviance fiscale dans les représentations des magistrats. Pour en comprendre les ressorts, il faut appréhender la régulation de cette délinquance, à la fois par rapport à la nature du délit commis et par rapport au statut social de la personne poursuivie (II). 


\section{Méthodologie de la recherche et présentation des matériaux}

Le présent article repose sur une recherche menée de 2012 à 2014 auprès de l'ensemble des acteurs impliqués dans la répression de la fraude fiscale en France ${ }^{2}$. Une campagne d'entretiens a été effectuée auprès des agents de l'administration fiscale, puis des magistrats de l'institution judiciaire, afin de mettre en lumière le rôle qu'ils jouent dans la mise en œuvre de la sanction. C'est cette deuxième phase de l'enquête qualitative qui est restituée ici. Nous avons pu rencontrer six procureurs spécialisés dans la délinquance économique et financière, ainsi que six magistrats du siège. Trois entretiens ont enfin été réalisés avec des membres des services d'enquête judiciaire, ainsi qu'avec un cadre du ministère de la Justice et trois avocats, issus des deux cabinets chargés de représenter l'administration fiscale.

Pour rendre compte des cas de fraude fiscale soumis à l'institution judiciaire et compléter les statistiques publiques, nous avons également constitué une base de données à partir des affaires terminées par un jugement ou un arrêt rendu en $2011^{3}$. Les décisions ainsi collectées sans biais - concernent 570 prévenus, auxquels il faut ajouter 23 personnes qui ont fait l'objet d'un non-lieu avant tout renvoi devant une juridiction de jugement. Plus de la moitié de ces jugements de première instance (330, soit 57,9\%) ont été rendus en 2011 ; ceux qui ont été rendus entre 2007 et 2010 figurent dans la base parce qu'ils ont été frappés d'appel, voire de pourvoi en cassation. Cette exploitation statistique nous a permis de nous approcher plus précisément du profil des contribuables mis en cause, notamment sur des variables non codées par la statistique publique (comme la nationalité, le lieu de naissance ou l'existence d'antécédents judiciaires), la nature des affaires soumises au juge et les types de sanctions adoptées par l'institution judiciaire à leur encontre.

2. Financée par la Mission de recherche Droit et Justice, cette recherche a donné lieu à un rapport intitulé «Des erreurs ou des fraudes? Le sens des sanctions en matière fiscale». Les résultats exposés ci-après reprennent, dans une large mesure, les thématiques développées dans L'impunité fiscale. Quand l'État brade sa souveraineté, Paris, La Découverte, 2015.

3. Nous remercions très vivement Maud Choquet qui a procédé au codage de ces jugements. 


\section{La place de la fraude fiscale dans le champ judiciaire}

En France, toute fraude fiscale supérieure à 153 euros peut en principe donner lieu à des poursuites pénales alors qu'en Espagne ou en Italie, un manque à gagner pour le Trésor public d'au moins 77000 ou 120000 euros est nécessaire. Mais cette rigueur hexagonale n'est qu'apparente; en pratique, les fraudes poursuivies sont triées sur le volet. L'institution judiciaire ne peut intervenir qu'à la suite d'une plainte du ministre du Budget. Chaque année, sur les 16000 dossiers pour lesquels un manquement volontaire a été constaté au cours du contrôle fiscal, l'administration des impôts sélectionne un peu plus d'un millier de dossiers examinés ensuite par la Commission des infractions fiscales (CIF) (composée de hauts fonctionnaires et de magistrats). Celle-ci a, en règle générale, fixé à 100000 euros le seuil minimal pour transmettre un dossier aux juges.

Comme le suggère la métaphore de l'entonnoir (Aubusson de Cavarlay, 1999), l'institution judiciaire n'intervient donc qu'après un long processus de filtrage. L'objectif revendiqué pour l'action pénale est d'ajouter aux sanctions administratives déjà prises une dimension sup-

\section{F I G URE 1}

\section{L'entonnoir de la régulation administrative des fraudes fiscales}

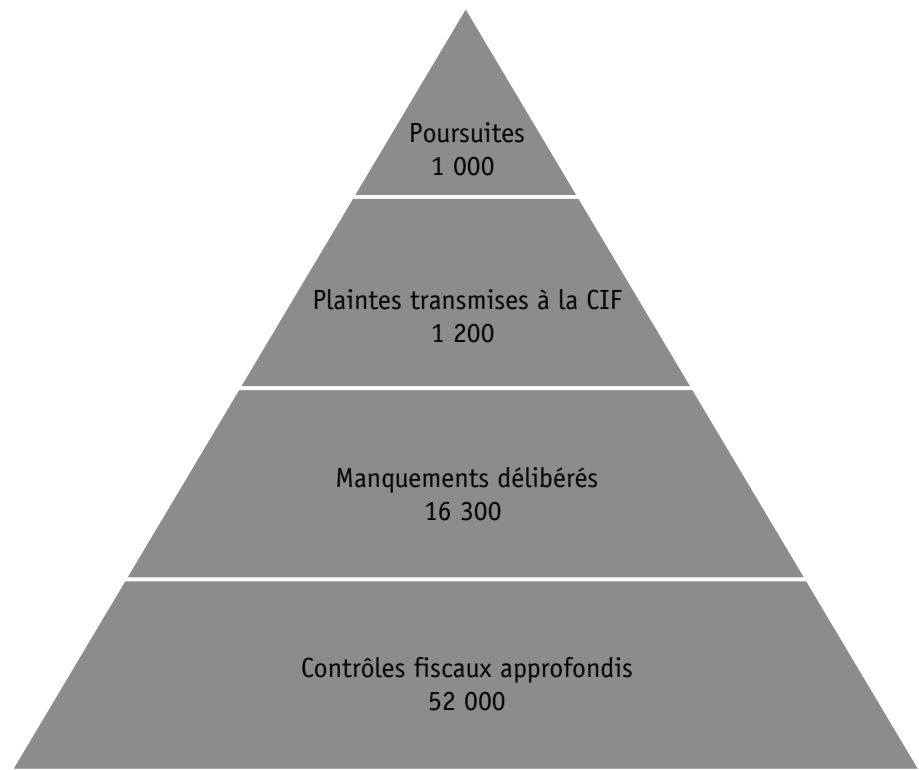

Source: Rapport de la Direction générale des finances publiques 2014 (chiffres 2013, arrondis). 
plémentaire, morale et dissuasive. Les magistrats que nous avons rencontrés s'approprient sans difficulté cette fonction; en paroles, ils partagent l'idée que les infractions fiscales les plus graves doivent donner lieu non seulement à des amendes administratives, mais aussi à une procédure ayant une connotation punitive plus nette. Mais en pratique, ils s'impliquent peu dans un travail de pénalisation qui a pour particularité de dépendre d'une autre institution - l'administration fiscale - dotée du pouvoir de sélectionner en amont les dossiers des contribuables susceptibles d'être sanctionnés.

\section{La fraude fiscale: un contentieux marginal de petits délinquants}

Les poursuites pénales pour fraude fiscale représentent une part très limitée (3\%) de la délinquance économique et financière qui elle-même occupe une position de relégation dans le champ judiciaire. Si près du quart des cas sont jugés à Paris, plus de la moitié des plaintes échouent dans d'autres tribunaux qui en traitent moins de dix par an et où les magistrats sont généralement peu formés au droit fiscal. La matière n'est pratiquement pas enseignée à l'École nationale de la magistrature et reste perçue comme technique et rébarbative par la plupart des magistrats qui s'y heurtent.

En principe, les procureurs de la République sont non seulement chargés de décider si tel ou tel délit doit être poursuivi, mais aussi de choisir la réponse pénale la plus appropriée (Milburn, Salas et Kotulski, 2010). Mais en matière de fraude fiscale, ils ne jouent pas, ou très peu, ce rôle de pilotage: ils doivent faire face à des affaires qui suivent un circuit très stéréotypé. Écartant systématiquement la comparution immédiate et le classement sans suite, ils peuvent, en théorie, demander la désignation d'un juge d'instruction qui dirigera des investigations approfondies. En pratique, il est cependant exceptionnel qu'une information judiciaire soit ouverte pour fraude fiscale:

Moi, en tant que juge d'instruction, je vois très peu de fraudes fiscales. Je ne peux pas les voir, parce que je n'en suis pas saisi, pour la simple et bonne raison que la plupart d'entre elles portent sur des infractions et sur des cas tellement simples que ça ne mérite pas une instruction...

Entretien avec un juge d'instruction spécialisé dans les affaires économiques et financières 
Comme le souligne ce juge d'instruction ayant 25 ans d'ancienneté, la nature des affaires sélectionnées par l'administration n'incite guère les magistrats à engager une enquête fouillée: les dossiers que leur transmet l'administration sont souvent ceux de gérants de petites et moyennes entreprises, impliqués dans des fraudes apparemment assez simples. Dans notre échantillon de 570 jugements, aucun dossier n'émane du service chargé du contrôle des plus grandes entreprises, alors que celuici notifie environ $20 \%$ du montant de tous les redressements répartis sur moins de 1500 dossiers, soit, en 2009, une dette fiscale moyenne de 2,5 millions d'euros par entreprise contrôlée (Cour des comptes, 2012). Si les grandes entreprises sont absentes des dossiers transmis par le fisc au juge pénal, ce n'est pas directement en raison d'une connivence avec le gouvernement en place ou parce qu'elles disposeraient d'un pouvoir politique (Coleman, 1985). C'est plutôt parce que l'objectif d'obtenir un recouvrement rapide et certain conduit les vérificateurs à privilégier les transactions et parce que ces sociétés savent donner à leurs fraudes l'apparence d'erreurs. Il en est de même pour les contribuables les plus fortunés et ceux disposant d'un gros patrimoine: les fraudes impliquant l'impôt de solidarité sur la fortune ou les droits sur les successions ne font jamais (ou presque) l'objet de poursuites ${ }^{4}$. Ainsi la fraude patrimoniale, c'est-à-dire celle liée à l'évaluation, la cession ou la transmission des patrimoines, échappe pratiquement à toute forme de pénalisation. Selon la Commission des affaires fiscales (2013), l'essentiel des poursuites concerne trois types d'impôts - la taxe sur la valeur ajoutée ( $53 \%$ des affaires), l'impôt sur les sociétés $(24 \%)$ et l'impôt sur le revenu $(22,5 \%)$ - et se concentre sur certains secteurs d'activité. Ainsi, en 2013, le secteur de la construction (bâtiments et travaux publics) arrive en tête avec $27 \%$ des poursuites, alors qu'il ne représente que $6 \%$ de la valeur ajoutée de l'ensemble des branches; les activités de sécurité privée sont à l'origine de presque $4 \%$ des poursuites, alors que leur poids dans l'économie n'est que de $0,3 \%$. À l'inverse, le secteur immobilier représente presque $13 \%$ du tissu fiscal, mais seulement $5 \%$ des fraudes portées devant un tribunal correctionnel. Derrière le poids des secteurs du bâtiment, du gardiennage et du

4. Aucun dossier n'apparaît dans notre base statistique; trois fraudes aux droits d'enregistrement et à l'impôt de solidarité sur la fortune ont été transmises à la Commission des infractions fiscales dans le cadre de la procédure classique en 2013 (cf. Commission des infractions fiscales, Rapport annuel à l'attention du gouvernement et du Parlement, 2013, p. 6). 
nettoyage apparaît également le critère de la nationalité: les étrangers constituent, dans notre base, $30 \%$ des délinquants convaincus de fraude fiscale alors qu'ils ne représentent que $6 \%$ de la population résidant en France et $13 \%$ des condamnés, toutes délinquances confondues 5 . Cette surreprésentation des étrangers, et notamment des Turcs, parmi les contribuables poursuivis pour fraude fiscale ne signifie pas qu'ils soient plus enclins à frauder, mais tient essentiellement à la simplicité des moyens qu'ils utilisent pour contourner l'impôt. Par méconnaissance ou méfiance, ils s'abstiennent de négocier avec l'administration fiscale et transgressent la règle de façon plus évidente que ceux qui savent en jouer. Ils se retrouvent ainsi plus souvent devant les juges qui, face à ces contribuables démunis ou absents, ont le sentiment de ne pas se trouver en présence des véritables fraudeurs:

La plupart des dossiers qui nous sont déposés ne correspondent pas à ce qu'on attend, nous, d'un dossier pénal... Je n'ai jamais compris, moi, ce qui motivait Bercy pour sélectionner les plaintes... La plupart des dossiers concernent des faits qui sont trop vieux et pour lesquels ensuite on n'a plus les moyens de retrouver les véritables auteurs... Dans les escroqueries en bande organisée, les escrocs utilisent des hommes de paille qu'ils vont instituer responsables légaux et lorsque le fisc ne poursuit que l'homme de paille, c'est tirer complètement à côté de la cible! Donc c'est ridicule et en terme pénal [sic], c'est inopérant, parce qu'on va poursuivre un type qui est garçon de café, clochard ou sans-papiers...

Entretien avec un procureur spécialisé dans les affaires économiques en région parisienne

Ce procureur déplore que ne lui soient déférés que les responsables apparents de la fraude (gérants de paille notamment). En fustigeant le monopole de l'administration fiscale pour le choix des dossiers à poursuivre, il justifie par ce biais la passivité de l'institution judiciaire. Mais il omet de reconnaitre que celle-ci renonce également le plus souvent à rechercher les véritables organisateurs de la fraude fiscale. Tout se passe comme si l'implication de différentes institutions dans la régulation des déviances fiscales permettait à chaque acteur de renvoyer la responsabilité de l'impunité sur les autres protagonistes.

5. Ministère de la Justice, Les condamnations en 2012, Sous-direction de la statistique et des études, 2013. 


\section{Des investigations a minima}

Les magistrats du parquet, qui pilotent la quasi-totalité des enquêtes de fraude fiscale, n'utilisent pas leurs pouvoirs de manière active. Les objectifs chiffrés auxquels ils sont soumis (Gautron, 2014) ne les incitent guère à regrouper des affaires qui pourraient être liées entre elles. Lorsque des entreprises associées à un même montage fiscal sont dispersées sur le territoire, les plaintes sont généralement déposées devant plusieurs tribunaux. Leur centralisation entre les mains d'un seul procureur est rare: la priorité est donnée à de petits dossiers susceptibles d'être bouclés rapidement plutôt qu'aux affaires d'envergure, aux ramifications complexes. Même la jonction entre la fraude fiscale et les délits connexes (tel l'abus de biens sociaux) commis par un même contribuable est rarement effectuée par les procureurs:

C'est nous [les magistrats du siège] qui faisons les regroupements par la mémoire que l'on peut avoir des dossiers... mais le procureur, lui, il ne le fait pas...

Entretien avec une magistrate du siège dans une chambre financière d'un tribunal en région parisienne

La quantification de l'activité au regard du nombre de dossiers traités conduit les magistrats à ne pas regrouper systématiquement les dossiers imbriqués, surtout lorsqu'ils sont répartis entre plusieurs juridictions.

Dans les affaires de fraude fiscale, le travail d'orientation des poursuites, qui constitue en principe une mission essentielle du ministère public, reste donc pratiquement inexploité. De manière quasi mécanique, le magistrat du parquet se contente de transmettre une demande d'enquête aux services de police ou de gendarmerie qui réalisent une enquête a minima. La garde à vue est très peu pratiquée, et quand elle intervient, elle excède rarement quelques heures. Lorsqu'au cours de son interrogatoire, la personne mise en cause se retranche derrière la responsabilité d'un gérant de fait ou d'un comptable, celui-ci est parfois entendu. Mais l'enquête est si sommaire qu'elle conduit rarement à découvrir d'autres coupables ou complices. Même confiées à des services spécialisés, les investigations peinent à réunir les preuves suffisantes pour mettre en cause les organisateurs ou les commanditaires de la fraude. Dans une affaire classique où un entrepreneur étranger a endossé une fraude fiscale commise (au moins en partie) par d'autres personnes, la présidente d'audience le déplore: «tant que les enquêteurs n'iront pas plus loin pour voir les entreprises qui sont au-dessus, on n'avancera 
pas»; mais le tribunal condamnera le prévenu sans ordonner de supplément d'enquête 6 .

Ainsi, la relative passivité des magistrats du parquet est le produit d'un ajustement entre les contraintes auxquelles ils sont soumis et leur propre perception du statut marginal de ce délit. Ils admettent d'autant mieux le désinvestissement de l'institution judiciaire qu'ils le perçoivent sans grande conséquence: le premier travail accompli par l'administration fiscale, en amont de la plainte, suffit généralement à établir la fraude et à éviter une relaxe.

Le traitement pénal de la fraude fiscale reste ainsi, encore aujourd'hui, le parent pauvre de la lutte contre la délinquance économique et financière. Le retentissement médiatique de plusieurs affaires de grande envergure (Cahuzac, HSBC) a contraint les pouvoirs publics à adopter de nouvelles mesures annoncées à grand renfort de communications. Les dossiers confiés, en amont du contrôle fiscal, au procureur de la République financier qui peut s'appuyer sur des services d'enquête spécialisés, ou ceux ouverts à l'initiative de magistrats (pour blanchiment de fraude fiscale) restituent un rôle actif à l'institution judiciaire. Mais l'attention portée à une centaine d'affaires par an ne doit pas faire oublier les conditions dans lesquelles sont instruits tous les autres dossiers de fraude fiscale. Dans la majorité des cas, les magistrats du parquet restent, sans en avoir toujours conscience, dépendants de la mise en forme opérée par l'administration fiscale. Dans un contexte de rareté des juges d'instruction financiers et des enquêteurs spécialisés, tout les incite à s'en tenir à l'apparence de «dossiers bouclés» et à ne procéder qu'à des investigations minimales.

\section{Articuler l'approche par les délits et par les délinquants}

La démobilisation observée chez les procureurs se retrouve également parmi les magistrats du siège. Dans leur très grande majorité, les affaires de fraude fiscale sont perçues comme inintéressantes et sont jugées en quelques dizaines de minutes. Pour d'autres types de délits, une telle rapidité d'examen conduit à des condamnations sévères (Saas, Lorvellec et Gautron, 2013), mais c'est le contraire qui se produit dans le domaine

6. Tribunal de grande instance de Paris, $11^{\mathrm{e}}$ chambre, audience du 13 février 2015, $13 \mathrm{~h} 30$. 
de la fraude fiscale. L'indulgence des juges peut d'abord s'expliquer par la nature singulière de ce délit. S'intéressant au cas de l'Allemagne des Trente Glorieuses, Schmölders (1973) justifiait cette moindre pénalisation par le fait que la fraude fiscale reste assimilée à une faute que tout le monde peut commettre et qui n'a rien à voir avec la moralité de la personne. Plus récemment, Levi (2010) a développé l'idée d'une impunité fiscale qui tiendrait à la position sociale de ceux qui cherchent à éviter l'impôt. Ces deux approches, l'une par le délit et l'autre par les délinquants (Lascoumes et Nagels, 2014), sont généralement présentées comme distinctes (Cusson, 1998). C'est pourtant leur combinaison qui permet de rendre compte d'une indulgence fonctionnant comme un inconscient d'institution.

\section{La mansuétude des juges à l'égard d'un délit singulier}

Alors que la législation française affiche des sanctions sévères (jusqu'à cinq, voire sept ans de prison), plus des trois quarts des fraudeurs du fisc traduits devant les tribunaux sont seulement condamnés à de la prison avec sursis (contre un tiers pour les auteurs de délits en général) pour une durée moyenne de neuf mois, et à une amende de quelques milliers d'euros. Seul un fraudeur sur dix est condamné à une peine de prison ferme, soit deux fois moins que pour l'ensemble des délits? En matière de fraude fiscale, les magistrats s'en tiennent ainsi au principe général, posé par le code pénal et répété par les circulaires ${ }^{8}$, selon lequel, en matière délictuelle, la prison ferme est un «ultime recours». Au-delà même du cas français, cette délinquance bénéficie d'un traitement judiciaire plus clément que celui réservé à la criminalité conventionnelle et même, d'une manière générale, à la criminalité en col blanc (Friedrichs, 2009).

En première analyse, la faible proportion de condamnations à des peines de prison ferme tient à la place qu'occupe le délit de fraude fiscale dans les représentations des juges. Évoquant le cas de pharmaciens poursuivis pour avoir utilisé un logiciel frauduleux permettant

7. Ces proportions sont celles qui ressortent de notre base statistique; elles correspondent à celles qui nous ont été communiquées par le ministère de la Justice à partir des inscriptions aux casiers judiciaires.

8. Article 132-24 du Code pénal; circulaire du 10 novembre 2010 relative à la présentation des dispositions de la loi pénitentiaire n 2009-1436 du 24 novembre 2009 relatives au prononcé des peines et aux aménagements de peines, NOR JUSD1028753C. 
d'occulter certaines recettes, ce magistrat du siège justifie en ces termes les décisions de relaxe et prison avec sursis que son tribunal a prononcées :

Quand on juge les affaires fiscales, on n'est pas là pour faire des exemples. On doit prendre en compte des faits de société et remettre les gens dans le droit chemin... Il faut avoir une vision de l'ensemble de la délinquance et se demander quelles sont les réponses sociales appropriées. Et dans ce type de délinquance, quelle est la réponse la plus adaptée?

Entretien avec un magistrat du siège en région parisienne

Chaque fois qu'ils replacent la fraude fiscale dans l'ensemble des délits passibles de poursuites pénales, les magistrats relativisent la nécessité d'une sanction exemplaire: en l'absence de victime immédiatement identifiable et incarnée, l'évitement de l'impôt apparaît toujours moins grave que les atteintes aux biens ou aux personnes comme les vols, les agressions ou les violences. Mais cette tendance à minorer la gravité de la délinquance fiscale ne s'explique pas seulement par la place qu'occupe ce délit dans l'arène judiciaire: elle dépend également du profil social du délinquant et de sa capacité à adapter son comportement aux attentes des magistrats.

\section{L'empathie des juges à l'égard des délinquants fiscaux bien conseillés}

Pour mesurer les facteurs pouvant expliquer le prononcé par les juges d'une peine de prison ferme, nous avons recouru à une régression logistique, à partir des variables disponibles dans les jugements. Si aucune corrélation statistique ne peut être observée entre le niveau des condamnations et les caractéristiques sociales des mis en cause - à l'exception du sexe -, l'importance de la trajectoire pénale du prévenu apparaît déterminante dans l'appréciation du juge. Le niveau de la peine est étroitement corrélé aux antécédents judiciaires, c'est-à-dire à l'existence, ou non, de condamnations pénales antérieures. En revanche, le prononcé d'une peine de prison ferme n'est pas corrélé au lieu de jugement.

La condamnation à de la prison ferme touche plus souvent les hommes que les femmes ${ }^{9}$. Il s'agit là d'un trait commun de la délinquance

9. Dans notre échantillon, $94,2 \%$ des condamnations à de la prison ferme visent des hommes et cette proportion se retrouve dans les statistiques du ministère de la Justice. 
TABLEAU 1

Déterminants de la condamnation à une peine de prison ferme

\begin{tabular}{|c|c|c|}
\hline Variables testées & Modalités & Rapport de cotes \\
\hline Sexe & $\begin{array}{l}\text { Homme } \\
\text { Femme }\end{array}$ & $\begin{array}{l}1 \\
0,3^{*}\end{array}$ \\
\hline Passé judiciaire & $\begin{array}{l}\text { Sans antécédents } \\
\text { Avec antécédents }\end{array}$ & $\begin{array}{l}1 \\
24,3^{* *}\end{array}$ \\
\hline Nationalité & $\begin{array}{l}\text { Française } \\
\text { Étrangère }\end{array}$ & $\begin{array}{l}1 \\
\text { Non significatif }\end{array}$ \\
\hline Lieu de naissance & $\begin{array}{l}\text { En France } \\
\hat{A} \text { l'étranger }\end{array}$ & $\begin{array}{l}1 \\
\text { Non significatif }\end{array}$ \\
\hline Secteur d'activité & $\begin{array}{l}\text { Autre que le bâtiment } \\
\text { Bâtiment }\end{array}$ & $\begin{array}{l}1 \\
\text { Non significatif }\end{array}$ \\
\hline Année de naissance & & Non significatif \\
\hline
\end{tabular}

* Significatif au seuil de $10 \%$; ${ }^{*}$ significatif au seuil de $1 \%$.

Lecture: un prévenu de sexe féminin a une probabilité d'être condamné à une peine de prison ferme 3 fois inférieure à un prévenu de sexe masculin ayant les mêmes caractéristiques (passé judiciaire, nationalité, lieu de naissance, année de naissance, secteur d'activité).

en col blanc (Daly, 1989). Le phénomène est encore accentué dans les cas où deux conjoints comparaissent pour fraude fiscale. Les juges sont souvent prompts à admettre l'innocence de l'épouse lorsqu'elle affirme, de manière plausible, ne pas «s'occuper des papiers». Dans plusieurs dossiers issus de la liste Falciani, des prévenues ont pu ainsi dresser leur portrait en parfaites ignorantes et être relaxées en se conformant au cliché de rôles sociaux genrés. À la femme, les enfants et la maison; à l'homme, la déclaration de revenus, explique l'une d'elles devant le tribunal correctionnel de Paris: «mon mari, il me dit seulement signe; ça vous paraît peut-être bizarre, mais c'est comme ça que ça fonctionne chez nous». Dans ces affaires, les magistrats ont privilégié une conviction retirée de l'audience - qui ne heurtait pas leurs représentations - sur la présence objective de signatures ou sur le ressenti qui avait pu être celui des enquêteurs ${ }^{10}$.

De façon plus significative, les peines de prison ferme sont corrélées aux antécédents judiciaires; par cette formule, on se réfère à un ensemble plus large que la récidive, laquelle vise seulement la réitération d'une infraction proche ou équivalente d'un délit précédemment

10. TGI Créteil, audience du 22 janvier 2015, 13 h 30; TGI Paris, $32^{\mathrm{e}}$ chambre, audience du 2 février 2015, 13 h 30. 
condamné. C'est le cas par exemple du gérant d'un cabaret oriental ayant fait l'objet d'une fermeture administrative en 2010 et d'une plainte pour fraude fiscale quelques mois plus tard (impliquant un préjudice d'un peu plus de 100000 euros pour l'État): ayant déjà été condamné pour des délits de contrefaçon et étant absent à l'audience, il écope alors de douze mois de prison dont six ferme.

En revanche, en l'absence d'antécédents judiciaires, la condamnation à une peine de prison ferme est très rare. Les magistrats ignorent généralement les précédents démêlés de l'intéressé avec le fisc. Même quand ils en sont informés, ils ne tiennent pas compte de ces procédures qui n'ont pas été portées devant un tribunal et qu'ils considèrent comme insuffisamment contradictoires. Ces prévenus, qui ont déjà été rappelés à l'ordre par l'administration mais qui, par le passé, ont su éviter les poursuites pénales, sont souvent issus des classes dominantes et sont particulièrement armés pour utiliser l'audience: ils savent convaincre qu'ils n'ont pas les traits des escrocs habituels, qu'ils sont bien insérés socialement et qu'ils ressemblent, à cet égard, à leurs juges, même s'ils appartiennent à des mondes différents (Roussel, 2002). Leurs avocats, souvent spécialisés, ont beau jeu de mettre en exergue la complexité technique de la loi et l'ignorance de leur client, pour introduire un doute dans l'esprit des juges quant à l'intentionnalité du délit. Pour peu qu'il sache répondre aux attentes du juge (Spire et Weidenfeld, 2011), le prévenu peut ainsi saisir l'occasion de l'audience pour se défaire de l'image d'un contribuable récalcitrant et arrogant.

À l'inverse, à l'égard des contribuables qui refusent, y compris devant le tribunal, de se conformer aux injonctions à payer, les juges hésitent moins à prononcer une sentence ferme. Ainsi de ce conseiller financier qui, devant le tribunal, a persisté à nier la dissimulation d'avoirs en Suisse et a écopé d'une peine de deux ans dont six mois ferme ${ }^{11}$.

Le type qui a une attitude de déni complet, on se dit qu'il va recommencer, il est tellement dans une logique qui est à l'encontre de ce qu'on pourrait souhaiter, on se dit qu'une peine d'emprisonnement va être dissuasive.

Entretien avec une magistrate du siège dans une chambre financière en région parisienne

Comme l'explique cette magistrate, la peine de prison ferme est la réponse judiciaire à ceux qui refusent la logique de l'institution. En

11. TGI Paris, $11^{\mathrm{e}}$ chambre, jugement du 10 juin 2014. 
définitive, l'enfermement reste une condamnation très minoritaire réservée aux contribuables qui ont déjà eu affaire à la justice ou qui refusent catégoriquement de «jouer le jeu». De plus, dans les rares affaires où les magistrats prononcent quelques mois de prison ferme, ils savent bien que cette condamnation sera neutralisée par le juge d'application des peines et ne conduira pas effectivement le délinquant vers l'établissement pénitentiaire.

Mais si les magistrats écartent presque systématiquement la prison ferme, ils n'utilisent pas davantage les autres sanctions disponibles. Là encore, le statut social du prévenu peut jouer un rôle important. L'éventualité de proposer un travail d'intérêt général aux coupables de délinquance économique a par exemple paru incongrue à tous les magistrats que nous avons interrogés:

Non, non! Les peines d'intérêt général, ça ne serait pas adapté»! [...] Sur le cas du médecin, on n'allait pas demander un travail d'intérêt général, alors qu'il continue à travailler et que justement son travail lui permet d'avoir des revenus, et après d'indemniser la partie civile! Voilà, c'est la logique. Donc, il vaut mieux avoir une peine pour ces infractions-là, pour les primo délinquants, euh... d'un an, voire deux ans avec sursis.

Entretien avec un procureur dans un pôle spécialisé sur les affaires économiques en province

Ah, sur le ton de la plaisanterie, on a évoqué le fait de condamner des présidents du CAC40 à des travaux d'intérêt général... je vois mal M. Sellière ou autre accepter un travail d'intérêt général, mettre la combinaison verte et puis aller balayer...

Entretien avec un responsable des affaires fiscales dans un parquet en région parisienne

On perçoit derrière ces propos l'idée qu'une peine aux vertus éducatives doit être réservée aux délinquants dont le statut social se situe en bas de la hiérarchie. Tout se passe comme si la nécessité de normaliser les comportements (Foucault, 1975) ne s'appliquait pas aux délinquants fiscaux bien insérés socialement. Même l'exemple de Silvio Berlusconi qui, en 2013, a obtenu la conversion de sa peine de prison en travail d'intérêt général n'a pas modifié cette appréciation.

La privation des droits civiques, civils ou de famille est également très rarement prononcée (moins de $5 \%$ des cas) et porte généralement sur des durées courtes (1 ou 2 ans). Là encore, les magistrats interrogés considèrent que la violence de cette condamnation n'est pas adaptée à 
la sanction de la fraude fiscale. Le souci de préserver l'intégration sociale du délinquant, notamment pour lui permettre de rembourser sa dette fiscale, apparaît plus clairement encore lorsqu'il s'agit d'inscrire la condamnation au casier judiciaire. Pour les fonctionnaires ou pour ceux qui exercent une profession réglementée, cette mention peut être lourde de conséquences. Mais dès lors que les sommes dues ont été payées, le droit à l'oubli, grâce à la dispense d'inscription, est souvent accordé par les magistrats pour ne pas porter préjudice à l'activité professionnelle des délinquants.

Ce qui les chagrine beaucoup ici, c'est l'inscription au casier judiciaire, le B2. Des fois, ils font appel seulement pour obtenir la dispense. Je crois, mais je ne suis pas juriste, que le tribunal ne peut pas dispenser, mais la Cour le peut. Je vous donne l'exemple de M. C., prof... Il a tout payé, il voulait filer doux. Mais non, il a été condamné. Il a fait appel pour obtenir la dispense. Et là, à la Cour, ils ont accepté. Faut dire que c'était mettre sa carrière en péril. Pour..., idem: c'est un haut gradé dans la Légion d'honneur et ça le préoccupait de garder une condamnation inscrite au B2.

Entretien avec un chef de brigade chargé du suivi pénal en province

Dès que l'inscription au casier judiciaire peut avoir une incidence directe et profonde, en fermant une voie professionnelle, ils accèdent donc assez facilement à la demande d'oubli de l'infraction.

L'étude empirique des pratiques des magistrats en matière de fraude fiscale fait apparaître un décalage net entre les discours de l'institution judiciaire et son fonctionnement quotidien. La réticence des juges à prononcer des peines significatives dans ce domaine tient à la fois au statut symbolique de ce délit et aux propriétés sociales de ceux qui le commettent. L'entonnoir institutionnel qui conduit à le sanctionner, dans l'immense majorité des cas, par des pénalités fiscales sans passage devant un tribunal, lui donne une apparence moins grave que les atteintes aux personnes ou même d'autres formes d'escroquerie. Par ailleurs, la capacité de certains prévenus à mobiliser leur capital (économique et social) pour se conformer aux attentes de l'institution judiciaire conduit les magistrats à considérer que la simple honte d'être poursuivi devant une juridiction correctionnelle constitue, en soi, une punition suffisante. 


\section{Conclusion}

La difficulté des magistrats à condamner les prévenus de statut social élevé pour fraude fiscale est ancienne et s'observe dans bien d'autres pays que la France. Évoquer cette forme d'impunité en termes d'inconscient d'institution ne signifie pas que les magistrats ne sont jamais conscients de leurs pratiques: il s'agit plutôt de souligner que leur disposition à adopter une posture véritablement répressive à l'égard de ce délit se heurte à des contraintes institutionnelles (comme l'impossibilité de choisir les contribuables à poursuivre, le temps qui sépare la découverte de l'infraction de la procédure judiciaire, ou des peines privilégiant la mise à l'écart du délinquant) qui les conduisent à s'abstenir de toute sanction significative. S'y ajoute la conviction - en partie inconsciente - que pour ce délit, l'objectif de récupérer l'argent éludé l'emporte sur toute autre considération morale ou répressive. Si l'on compare la persistance de cette impunité fiscale avec l'évolution récente de la répression d'autres délits, on perçoit que le redéploiement du pouvoir pénal prend deux formes bien distinctes, selon les groupes sociaux et les situations auxquelles il s'applique.

Dans le domaine de la sécurité publique et de la petite délinquance, l'inflation pénitentiaire se présente comme la réponse adéquate à la menace que chaque transgression fait peser sur l'ordre social (Wacquant, 2009). Allant bien au-delà de l'objectif de dissuader les individus, la punition mobilise dans ce cas un sens collectif et remplit des fonctions d'ordre symbolique et idéologique. À l'inverse, à l'égard de la fraude fiscale - comme d'ailleurs pour d'autres formes de délinquance économique et financière -, le pouvoir pénal de l'État se comprend à l'aune exclusive de l'équation budgétaire et se trouve dépourvu de toute dimension morale. Les peines prononcées ne visent ni à dissuader ni à signifier une quelconque réprobation publique. Le caractère extrêmement limité de la publicité organisée par les pouvoirs publics français à l'encontre des personnes condamnées pour fraude fiscale montre bien qu'il ne s'agit pas de mettre en scène une condamnation sociale. Alors que la répression de la petite délinquance fait l'objet d'une communication incessante pour relayer les procès et les opérations de police, la théâtralisation de la lutte contre la fraude fiscale s'est arrêtée à l'enceinte parlementaire et aux discours politiques. 


\section{Références}

Aubusson de Cavarlay, B. (1999). Du dossier de procédure aux filières pénales, l'effet d'entonnoir et le syndrome du réverbère. Dans C. Faugeron (dir.), Les drogues en France: politiques, marchés, usages (p. 151-159). Genève, Suisse: Georg Editeur.

Bourdieu, P. (1980). Le mort saisit le vif. Les relations entre l'histoire réifiée et l'histoire incorporée. Actes de la recherche en sciences sociales, 32(1), 3-14.

Braithwaite, J. (1989). Crime, Shame and Reintegration. Cambridge, Royaume-Uni: Cambridge University Press.

Chauvin, S. et Jounin, N. (2011). L'externalisation des illégalités. Ethnographies des usages du travail 'temporaire' à Paris et à Chicago. Dans L. Fontaine et F. Weber (dir), Les paradoxes de l'économie informelle. À qui profitent les règles? (p. 113-138). Paris, France: Karthala.

Coleman, J. W. (1985). Law and power: The Sherman Antitrust Act and its enforcement in the petroleum industry. Social Problems, 32(3), 264-274.

Coleman, J. W. (1994). The criminal elite: Understanding white-collar crime. New York, NY: St Martin's Press.

Commission des infractions fiscales. (2013). Rapport annuel à l'attention du gouvernement et du parlement.

Cook, D. (1989). Rich Law, Poor Law. Differential Response to Tax and Supplementary Benefit Fraud. Milton Keynes, Royaume-Uni: Open University Press.

Cour des comptes. (2012). Le pilotage du contrôle fiscal. Rapport public annuel. Paris, France.

Cusson, M. (1998). Criminologie actuelle. Paris, France: Presses Universitaires de France.

Daly, K. (1989). Gender and varieties of white-collar crime. Criminology, 27(4).

Foucault, M. (1975). Surveiller et Punir. Paris, France: Gallimard.

Friedrichs, D. O. (2009). Trusted Criminals: White Collar Crime in Contemporary Society. Belmont, CA: Wadsworth.

Gautron, V. (2014). L'impact des préoccupations managériales sur l'administration locale de la justice pénale française. Champ pénal, 11.

Katz, J. (1979). Legality and Equality: Plea Bargaining in the Prosecution of White-Collar and Common Crimes. Law E Society Review, 13(2), 431-459.

Klepper, S. et Nagin, D. S. (1989). The deterrent effect of perceived certainty and severity of punishment revisited. Criminology, 27(4), 721-746.

Lascoumes, P. (1986). Les affaires ou l'art de l'ombre. Paris, France: Le Centurion.

Lascoumes, P. et Nagels, C. (2014). Sociologie des élites délinquantes: de la criminalité en col blanc à la corruption politique. Paris, France: Armand Colin.

Leroy, M. (2010). L'impôt, l'État et la société. Paris, France: Economica.

Levi, M. (2010). Serious tax fraud and noncompliance. Criminology $\mathfrak{F}$ Public Policy, 9(3), 493-513.

McBarnet, D. (1991). Whiter than white collar crime: tax, fraud insurance and the management of stigma. Britannic Journal of Sociology, 42(3), 323-344. 
Milburn, P., Salas, D. et Kotulski, K. (2010). Les procureurs: entre vocation judiciaire et fonctions politiques. Paris, France: PUF.

Murphy, K. (2008). Enforcing Tax Compliance: To Punish or Persuade? Economic analysis er Policy, 38(1), 113-135.

Nagels, C. (2013). Les grandes entreprises et les instances de lutte contre la fraude sociale: le jeu du chat et de la souris. Champ pénal, 10.

Roussel, V. (2002). Affaires de juges. Les magistrats dans les scandales politiques en France. Paris, France: La Découverte.

Saas, C., Lorvellec, S. et Gautron, V. (2013). Les sanctions pénales, une nouvelle distribution. Dans J. Danet (dir.), La réponse pénale. Dix ans de traitement des délits (p. 159-210). Rennes, France: Presses universitaires de Rennes.

Schmölders, G. (1973). Psychologie des finances et de l'impôt. Paris, France: Presses universitaires de France.

Shover, N. et Hochstetler, A. (2005). Choosing white-collar crime. New York, NY: Cambridge University Press.

Simpson, S. (2002). Corporate Crime, Law, and Social Control. New York, NY: Cambridge University Press.

Spire, A. (2011). La domestication de l'impôt par les classes dominantes. Actes de la recherche en sciences sociales, 190(5), 58-71.

Spire, A. et Weidenfeld, K. (2011). Le tribunal administratif: une affaire d'initiés? Les inégalités d'accès à la justice et la distribution du capital procédural. Droit et Société, 79(3), 689-713.

Spire, A. et Weidenfeld, K. (2015). L'impunité fiscale. Paris, France: La Découverte.

Sutherland, E. H. (1983). White collar crime: The uncut version. New Haven, CT: Yale University Press.

Wacquant, L. (2009). Punishing the Poor: The Neoliberal Government of Social Insecurity. Durham, NC: Duke University Press.

Weidenfeld, K. (2010). À l'ombre des niches fiscales. Paris, France: Economica.

\title{
Judicial attitudes towards tax fraud: the unconscious at work in institutions
}

\begin{abstract}
French criminal courts often deal leniently with tax fraud cases, but the individuals involved in handling such cases are not fully aware that this is the case. To analyze this collective institutional obliviousness, we look at the discourse and practice of prosecutors and judges working with tax fraud files passed on to them by the tax authorities. Although all these actors are convinced that the fight against this form of white-collar crime is necessary, they refrain from using all the means in their power to secure effective penalties. The result is an indulgent attitude that contrasts with the courts' intransigence in other fields. In order to get a clearer view of the fiscal impunity that results, we propose an approach that takes into account the uniqueness of this crime and these criminals, keeping in mind the legal and symbolic constraints affecting judges.
\end{abstract}

KEYWORDS - Tax fraud, white collar crime, sociology of law, judicial institution, norms. 


\section{La tolerancia de los jueces al fraude fiscal: un inconsciente institucional}

RESUMEN - La presente contribución busca demostrar que la regulación del fraude fiscal realizada por la institución judicial refleja una tolerancia, en parte inconsciente, de los actores a cargo. Para analizar sus mecanismos, el interés se centra sobre el discurso y las prácticas de los procuradores y magistrados encargados de asegurar el seguimiento penal de los casos que la administración fiscal les confía. Estos actores dicen estar convencidos de la necesidad de luchar contra esta forma de delincuencia de cuello blanco, pero se abstienen de utilizar todos los medios disponibles para que la penalización del fraude fiscal se concrete en sanciones efectivas. Resulta de ello una indulgencia que contrasta con la postura de intransigencia adoptada por la institución judicial en otras áreas. Para entender los alcances de esta impunidad fiscal, proponemos un enfoque que tiene en cuenta la singularidad de este delito y el perfil social de aquellos que lo cometen, considerando a la vez las restricciones institucionales que se imponen a los magistrados y las representaciones que los vuelven aceptables.

PALABRAS CLAVE - Fraude fiscal, delincuencia de cuello blanco, sociología del derecho, institución judicial, normas. 